hep-ph/9502418, WIS-95/7/Mar-PH

\title{
Lepton Mass Matrix Models
}

\author{
Yuval Grossman and Yosef Nir \\ Department of Particle Physics \\ Weizmann Institute of Science, Rehovot 76100, Israel
}

The smallness and hierarchy in fermion parameters could be the result of selection rules due to an Abelian horizontal symmetry broken by a small parameter. When applied to the lepton sector, then for a large class of models, a number of interesting order of magnitude relations arise: with $i<j, m\left(\nu_{i}\right) / m\left(\nu_{j}\right) \sim \sin ^{2} \theta_{i j} ; m\left(\ell_{i}^{-}\right) / m\left(\ell_{j}^{-}\right) \lesssim \sin \theta_{i j}$; $m\left(\nu_{i}\right) / m\left(\nu_{j}\right) \gtrsim m^{2}\left(\ell_{i}^{-}\right) / m^{2}\left(\ell_{j}^{-}\right) ; m\left(\nu_{e}\right) \lesssim m\left(\nu_{\mu}\right) \lesssim m\left(\nu_{\tau}\right)$. The relations between neutrino masses and mixings may become exact if the horizontal symmetry together with holomorphy induce certain zero entries in the lepton mass matrices. A full high energy theory is likely to include scalars with flavor changing couplings and heavy leptons in vector representations; however, the masses of these particles are too heavy to be directly observed in experiment. Indirect evidence for the horizontal symmetry may arise from other sectors of the theory: non-degenerate sleptons are allowed as the symmetry aligns lepton and slepton mass matrices; light leptoquarks are allowed as the symmetry can make their couplings diagonal and chiral. 


\section{Introduction}

Only three parameters of the lepton sector are unambiguously experimentally determined. These are the three charged lepton masses [1]:

$$
m_{e}=0.5110 \mathrm{MeV}, \quad m_{\mu}=105.7 \mathrm{MeV}, \quad m_{\tau}=1777 \mathrm{MeV} .
$$

The unambiguous information on neutrino masses consists of only upper bounds [1]:

$$
m_{\nu_{e}} \leq 5.1 \mathrm{eV}, \quad m_{\nu_{\mu}} \leq 0.16 \mathrm{MeV}, \quad m_{\nu_{\tau}} \leq 31 \mathrm{MeV} .
$$

However, various theoretical arguments and observational puzzles suggest that neutrinos may have non-vanishing masses. For example, if $\nu_{\tau}$ is to be an important component in the dark matter then

$$
m_{\nu_{\tau}}=\mathcal{O}(10 \mathrm{eV}) .
$$

If the solar neutrino problem is solved by the MSW mechanism with $\nu_{e}-\nu_{\mu}$ oscillations then [2] most likely,

$$
m_{\nu_{\mu}}^{2}-m_{\nu_{e}}^{2} \sim 6 \times 10^{-6} e V^{2}, \quad \sin ^{2} 2 \theta_{12} \sim 7 \times 10^{-3} .
$$

In any case, the lepton parameters (certainly the charged lepton masses and very likely also mixing angles and neutrino masses) are small and hierarchical.

The smallness and hierarchy are the most puzzling features of fermion parameters. They suggest that there should exist a more fundamental theory where the hierarchy is generated in a natural way. Once such a framework is found, we should investigate its predictions and find ways in which it can be experimentally tested. Such a task, based on the wealth of information on the quark sector, was recently taken in refs. [3] [4] [5]. The smallness and hierarchy of the quark masses and mixing angles can be naturally generated in theories which, at low energy, are described effectively by an Abelian horizontal symmetry that is explicitly broken by a small parameter. The possible evidence for such a symmetry divides into three types:

(i) Numerology: the symmetry leads to order of magnitude relations among the various parameters, e.g. $\left|V_{u b}\right| \sim\left|V_{u s} V_{c b}\right|$. 
(ii) Direct evidence: the full high energy theory is likely to require the existence of new particles, e.g. scalars with flavor changing couplings and quarks in vector representations.

(iii) Indirect evidence: the symmetry has implications for other sectors of the theory, e.g. the squark spectrum [4], the Higgs potential [5], leptoquark couplings [6] and baryon number violation [7] [8].

The conclusion of ref. [5] was that it is the indirect evidence that is most likely to reveal the existence of the horizontal symmetry mechanism. In this work we extend the study of refs. [3] [4] 60 to the lepton sector. (For recent, related work, see refs. 99] [10].) Even though there is much less experimental information in the lepton sector, we are able to find interesting predictions that are independent of details of the lepton spectrum.

The paper is organized as follows: in section 2 we present the theoretical low energy framework in which we work, namely the lepton and scalar fields, the symmetry and the selection rules. In section 3 we study features of the lepton parameters that generally follow from the selection rules and are independent of any specific phenomenological input. In section 4 we make some assumptions about neutrino masses and mixing and present explicit models where the resulting hierarchy in lepton parameters arises naturally. In section 5 we investigate the possibility of exact relations among lepton parameters. Section 6 studies the embedding of the low energy effective model in a full high energy theory and the constraints on the relevant energy scales. In section 7 we investigate whether leptonslepton alignment could solve the problem of unacceptably large lepton flavor changing processes without slepton degeneracy. In section 8 we investigate the role of horizontal symmetries in allowing for light leptoquarks. In section 9 , the possibility of an unbroken horizontal symmetry is explored. Our conclusions are summarized in section 10.

\section{The Theoretical Framework}

We work in the framework of supersymmetric Abelian horizontal symmetry that has been recently investigated in refs. [3] [4] [5]. We assume that the low energy spectrum consists of the fields of the minimal supersymmetric Standard Model. The lepton and 
Higgs supermultiplets carry the following $S U(2)_{L} \times U(1)_{Y}$ quantum numbers:

$$
L_{i}(2)_{-1 / 2}, \quad \bar{\ell}_{i}(1)_{+1}, \quad \phi_{u}(2)_{+1 / 2}, \quad \phi_{d}(2)_{-1 / 2}
$$

where $i=1,2,3$ is a generation index. The $L_{i}$ fields are distinguished from the $\phi_{d}$ field by $R$ parity $\left(R_{p}\right)$ : in $L_{i}$, the fermion components are $R_{p}$ even, while in $\phi_{d}$ the scalar component is $R_{p}$ even. Each of the supermultiplets in (2.1) carries a charge under an Abelian horizontal symmetry $\mathcal{H}$. For most of our discussion, it makes no difference whether $\mathcal{H}$ is local or global, continuous or discrete. (For a discrete $Z_{N}$, we usually assume that $N$ is large enough that the Yukawa sector has an effective $U(1)$ symmetry.) $\mathcal{H}$ is explicitly broken by a small parameter $\lambda$ to which we attribute charge -1 . Then, the following selection rules apply:

a. Terms in the superpotential that carry charge $n \geq 0$ under $\mathcal{H}$ are suppressed by $\mathcal{O}\left(\lambda^{n}\right)$, while those with $n<0$ are forbidden due to the holomorphy of the superpotential. (If $\mathcal{H}=Z_{N}$, the suppression is by $\mathcal{O}\left(\lambda^{n(\bmod N)}\right)$.)

b. Terms in the Kähler potential that carry charge $n$ under $\mathcal{H}$ are suppressed by $\mathcal{O}\left(\lambda^{|n|}\right)$ $\left(\operatorname{or} \mathcal{O}\left(\lambda^{\min [ \pm n(\bmod N)]}\right)\right.$ for $\left.\mathcal{H}=Z_{N}\right)$.

Explicitly, the lepton parameters arise from the Yukawa terms

$$
Y_{i j} L_{i} \bar{\ell}_{j} \phi_{d}+\frac{Z_{i j}}{M_{L}} L_{i} L_{j} \phi_{u} \phi_{u} .
$$

$Y_{i j}$ is a generic complex $3 \times 3$ matrix that gives masses to the charged leptons. $Z_{i j}$ is a symmetric complex $3 \times 3$ matrix that gives Majorana masses to the neutrinos. $M_{L}$ is a high energy scale.

The first selection rule gives

$$
\begin{gathered}
H\left(L_{i}\right)+H\left(\bar{\ell}_{j}\right)+H\left(\phi_{d}\right)=n \Longrightarrow Y_{i j}= \begin{cases}\mathcal{O}\left(\lambda^{n}\right) & n \geq 0, \\
0 & n<0 .\end{cases} \\
H\left(L_{i}\right)+H\left(L_{j}\right)+2 H\left(\phi_{u}\right)=m \Longrightarrow Z_{i j}= \begin{cases}\mathcal{O}\left(\lambda^{m}\right) & m \geq 0, \\
0 & m<0 .\end{cases}
\end{gathered}
$$

If the sum of charges is non-integer, the corresponding coupling vanishes.

The second selection rule has implications for the potential renormalization of the kinetic terms. The canonical terms may be modified to

$$
\sum_{\ell, i, j} R_{i j}^{\ell} \ell_{i}^{\dagger} \gamma^{\mu} \partial_{\mu} \ell_{j}
$$


where $\ell=L, \bar{\ell}$ and $i, j=1,2,3$. Then

$$
H\left(\ell_{i}\right)-H\left(\ell_{j}\right)=k \Longrightarrow R_{i j}^{\ell}=\mathcal{O}\left(\lambda^{|k|}\right)
$$

The subtleties that arise when the kinetic terms are renormalized were investigated in ref. [5]. It was proved that:

1. The coefficients of order one may change, but the order of magnitude estimates in (2.3) and (2.4) remain valid.

2. The zeros in the Yukawa matrices may be lifted, but the corresponding entries are still highly suppressed and do not affect our results.

When discussing the lepton Yukawa couplings, we can set two of the relevant $H$ charges to zero. We can always use $U(1)_{Y}$ to set $H\left(\phi_{u}\right)=0$. As (2.2) is $U(1)_{X}$ invariant $\left(X\left(\phi_{d}\right)=-1, X\left(\bar{\ell}_{i}\right)=+1, X\left(L_{i}\right)=X\left(\phi_{u}\right)=0\right)$, we can set $H\left(\phi_{d}\right)=0$ without affecting our analysis of the Yukawa sector. Note also that neutrino masses depend on the unknown scale $M_{L}$. In models where all $H\left(L_{i}\right)$ are positive, we will use a modified scale, $\tilde{M}=$ $\frac{M_{L}}{\lambda^{2 H\left(L_{3}\right)}}$, and modify the charge to $H\left(L_{3}\right)=0$.

Any horizontal symmetry that acts on the quark sector has to be completely broken: an unbroken horizontal symmetry leads to either degenerate quarks or vanishing mixing angles [11][3]. Both possibilities are experimentally excluded. On the other hand, as neither neutrino masses nor lepton mixing angles have been unambiguously measured, the possibility of an exact horizontal symmetry (acting non-trivially in the lepton sector only) remains open. In most of this paper we assume that $\mathcal{H}$ is completely broken, namely that all lepton fields carry integer $H$-charges. We discuss the possibility of an unbroken horizontal symmetry in section 9 .

\section{General Results}

The special form of the neutrino Yukawa matrices (2.2), which has no analogue in the charged fermion sectors, has many interesting consequences. In particular, we find that as far as "numerology" is concerned, our framework is much more predictive in the neutrino sector than in any other sector. 
There are nine independent physical parameters (ignoring CP-violation): three mixing angles, three neutrino masses and three charged lepton masses. However, as explained in the previous section, there are only six relevant $H$-charges. Therefore, our framework predicts three order of magnitude relations among the physical parameters independent of specific charge assignments. 1 These relations may involve the neutrino masses and the mixing angles but not the charged lepton masses. The reason is that the six mixing angles and neutrino masses depend on the three $H\left(L_{i}\right)$ only, while the three charged lepton masses depend also on the three $H\left(\bar{\ell}_{i}\right)$.

To find the three relations, we note that the selection rules given in the previous section allow an order of magnitude estimate for the various mass ratios and mixing angles. With the choice $H(\lambda)=-1$, in most of our models all lepton charges are positive, $H\left(L_{i}\right), H\left(\bar{\ell}_{i}\right) \geq$ 0 . Then, ordering the lepton fields such that, for $i<j, H\left(L_{i}\right) \geq H\left(L_{j}\right)$ and $H\left(\bar{\ell}_{i}\right) \geq H\left(\bar{\ell}_{j}\right)$, we get

$$
\begin{aligned}
\sin \theta_{i j} & \sim \lambda^{H\left(L_{i}\right)-H\left(L_{j}\right)}, \\
\frac{m_{\nu_{i}}}{m_{\nu_{j}}} & \sim \lambda^{2\left[H\left(L_{i}\right)-H\left(L_{j}\right)\right]}, \\
\frac{m_{\ell_{i}}}{m_{\ell_{j}}} & \sim \lambda^{H\left(L_{i}\right)+H\left(\bar{\ell}_{i}\right)-H\left(L_{j}\right)-H\left(\bar{\ell}_{j}\right)} .
\end{aligned}
$$

In some of our models, some lepton fields carry negative $H$ charges. In these models, holomorphy plays an important role and the naive estimates (3.1) may be violated. Consequently, the relations given below do not hold in these models and other, model-specific relations replace them. The three relations can be easily found from Eq. (3.1):

$$
\begin{aligned}
& \frac{m_{\nu_{e}}}{m_{\nu_{\mu}}} \sim \sin ^{2} \theta_{12}, \\
& \frac{m_{\nu_{\mu}}}{m_{\nu_{\tau}}} \sim \sin ^{2} \theta_{23}, \\
& \frac{m_{\nu_{e}}}{m_{\nu_{\tau}}} \sim \sin ^{2} \theta_{13} .
\end{aligned}
$$

In other words, given, say, a single mass ratio in the neutrino sector and a single (independent) mixing angle, our framework predicts the order of magnitude of all other neutrino mass ratios and mixing angles.

1 This situation should be compared to the quark sector, where there is a single such relation, $\left|V_{u b}\right| \sim\left|V_{u s} V_{c b}\right|$. 
Examining Eqs. (3.1) we further find2

$$
\sin \theta_{i j} \gtrsim \frac{m_{\ell_{i}}}{m_{\ell_{j}}}
$$

As lepton masses are known, we can predict

$$
\begin{aligned}
& \sin \theta_{12} \gtrsim 0.002, \\
& \sin \theta_{23} \gtrsim 0.03, \\
& \sin \theta_{13} \gtrsim 0.0001 .
\end{aligned}
$$

(To estimate these lower bounds, we take $\frac{1}{2} \frac{m_{\ell_{i}}}{m_{\ell_{j}}}$ to allow for the uncertainty in the order of magnitude estimates.) Particularly encouraging is the rather large value of $\sin \theta_{23}$ which, if $m_{\nu_{\tau}}$ is in the appropriate range, would allow the detection of $\nu_{\mu}-\nu_{\tau}$ oscillations in the CHORUS, NOMAD and E803 experiments. Actually, this mixing angle should be rather close to the experimental upper bound (for "large $\Delta m_{23}^{2}$ ") [1]: $\sin ^{2} 2 \theta_{23} \lesssim 4 \times 10^{-3}$.

Eqs. (3.1) also imply (this is equivalent to the combination of (3.2) and (3.3))

$$
\frac{m_{\nu_{i}}}{m_{\nu_{j}}} \gtrsim\left(\frac{m_{\ell_{i}}}{m_{\ell_{j}}}\right)^{2}
$$

We can predict then

$$
\begin{aligned}
& \frac{m_{\nu_{e}}}{m_{\nu_{\mu}}} \gtrsim 1 \times 10^{-5}, \\
& \frac{m_{\nu_{\mu}}}{m_{\nu_{\tau}}} \gtrsim 2 \times 10^{-3}, \\
& \frac{m_{\nu_{e}}}{m_{\nu_{\tau}}} \gtrsim 4 \times 10^{-8} .
\end{aligned}
$$

(To estimate these lower bounds, we take $\frac{1}{2} \frac{m_{\ell_{i}}^{2}}{m_{\ell_{j}}^{2}}$ to allow for the uncertainty in the order of magnitude estimates.) The prediction (3.5) has further interesting implications. It coincides with the "reasonable see-saw" assumption made in ref. [12]. In combination with cosmological constraints, it led to the conclusion that all neutrinos are likely to be lighter than $\mathcal{O}(100 \mathrm{eV})$.

2 Similar relations should hold for quarks, namely $\frac{m_{u_{i}}}{m_{u_{j}}}, \frac{m_{d_{i}}}{m_{d_{j}}} \lesssim\left|V_{i j}\right|$. It is encouraging that indeed all quark mass ratios fulfill these inequalities. 
Finally, (3.1) implies that the hierarchy in the neutrino sector is the same as in the charged lepton sector, namely

$$
m_{\nu_{e}} \lesssim m_{\nu_{\mu}} \lesssim m_{\nu_{\tau}}
$$

Here, $\nu_{e}, \nu_{\mu}, \nu_{\tau}$ denote the mass eigenstates with mixing of $\mathcal{O}(1)$ with $e, \mu, \tau$, respectively. 3

\section{Explicit Examples}

For the purposes of this section, we will take the mass of $\nu_{\tau}$ as given in (1.3), and the mass of $\nu_{\mu}$ and the $\nu_{e}-\nu_{\mu}$ mixing angle as given in (1.4). The charged lepton masses are given in (1.1). As we are interested in explaining the orders of magnitude of the various parameters and not in the exact numbers of order one, we will estimate the various mass ratios and mixing angles as approximate powers of a small parameter $\lambda$, where

$$
\lambda \sim 0.2
$$

as follows from the value of the Cabibbo angle in the quark sector. Explicitly:

$$
\begin{gathered}
\frac{m_{e}}{m_{\mu}} \sim \lambda^{3}, \quad \frac{m_{\mu}}{m_{\tau}} \sim \lambda^{2}, \\
\frac{m_{\nu_{\mu}}}{m_{\nu_{\tau}}} \sim \lambda^{5 \pm 1}, \quad \sin \theta_{12} \sim \lambda^{2} .
\end{gathered}
$$

We will further assume that $\tan \beta \equiv \frac{\left\langle\phi_{u}\right\rangle}{\left\langle\phi_{d}\right\rangle} \sim 1$ and, consequently, $\frac{m_{\tau}}{\left\langle\phi_{d}\right\rangle} \sim \lambda^{3}$. It is simple to modify our models to the case of large $\tan \beta$.

In the simplest case, the horizontal symmetry and the charge of the breaking parameter are

$$
\mathcal{H}=U(1), \quad H(\lambda)=-1
$$

As explained above, the input data in (4.2) and (4.3) are enough to fix the $H$-charges of all the relevant fields and, therefore, predict the order of magnitude of all remaining lepton parameters. If $m_{\nu_{\mu}} / m_{\nu_{\tau}} \sim \lambda^{5}$, there is an unbroken horizontal $Z_{2}$ symmetry, $\tau$-parity. We discuss this case separately in section 9 .

3 Again, a similar result should hold for quarks and it does: the CKM matrix is close to a unit matrix when we order the up and down mass eigenstates in the same order of masses. 
4.1. $m_{\nu_{\mu}} / m_{\nu_{\tau}} \sim \lambda^{4}$

The hierarchy in Eqs. (4.2) and (4.3) determines a unique set of $H$ charge assignments:

$$
\begin{array}{cccccccc}
L_{1} & L_{2} & L_{3} & \bar{\ell}_{1} & \bar{\ell}_{2} & \bar{\ell}_{3} & \phi_{u} & \phi_{d} \\
(4) & (2) & (0) & (4) & (3) & (3) & (0) & (0) .
\end{array}
$$

The lepton mass matrices have then the following form:

$$
M^{\ell} \sim\left\langle\phi_{d}\right\rangle\left(\begin{array}{ccc}
\lambda^{8} & \lambda^{7} & \lambda^{7} \\
\lambda^{6} & \lambda^{5} & \lambda^{5} \\
\lambda^{4} & \lambda^{3} & \lambda^{3}
\end{array}\right), \quad M^{\nu} \sim \frac{\left\langle\phi_{u}\right\rangle^{2}}{\tilde{M}}\left(\begin{array}{ccc}
\lambda^{8} & \lambda^{6} & \lambda^{4} \\
\lambda^{6} & \lambda^{4} & \lambda^{2} \\
\lambda^{4} & \lambda^{2} & 1
\end{array}\right) .
$$

We emphasize that, here and below, the sign " $~$ " implies that we only give the order of magnitude of the various entries; there is an unknown (complex) coefficient of $\mathcal{O}(1)$ in each entry that we do not write explicitly. Eq. (4.6) predicts

$$
\sin \theta_{23} \sim \lambda^{2}, \quad \sin \theta_{13} \sim \lambda^{4}, \quad m_{\nu_{e}} / m_{\nu_{\mu}} \sim \lambda^{4},
$$

consistent with (3.2). Note, in particular, that

$$
m_{\nu_{\tau}}^{2}-m_{\nu_{\mu}}^{2} \sim 100 \mathrm{eV}^{2}, \quad \sin ^{2} 2 \theta_{23} \sim 0.006
$$

which is of the order of present bounds.

4.2. $m_{\nu_{\mu}} / m_{\nu_{\tau}} \sim \lambda^{6}$

This is an example of a model where

$$
\frac{m_{\nu_{\mu}}}{m_{\nu_{\tau}}}<\left(\frac{m_{\mu}}{m_{\tau}}\right)^{2}
$$

This violates the naive prediction (3.5). As explained in section 3, (4.9) can only be accommodated in models where some of the horizontal charges are negative and holomorphy then gives zero entries in the mass matrices. Specifically, for the case under study, $M_{32}^{\ell}=0$ is required, so that at least one of the $H_{i}$-charges has to fulfill $H_{i}\left(L_{3}\right)+H_{i}\left(\bar{\ell}_{2}\right)<0$.

As a specific example we take

$$
H=U(1)_{H_{1}} \times U(1)_{H_{2}} ; \quad \lambda_{1}(-1,0) \sim \lambda, \quad \lambda_{2}(0,-1) \sim \lambda^{2} .
$$


Take the following set of $\left(H_{1}, H_{2}\right)$ charges:

$\begin{array}{cccccccc}L_{1} & L_{2} & L_{3} & \bar{\ell}_{1} & \bar{\ell}_{2} & \bar{\ell}_{3} & \phi_{u} & \phi_{d} \\ (1,2) & (3,0) & (0,0) & (-1,2) & (-2,2) & (1,1) & (0,0) & (0,0) .\end{array}$

The lepton mass matrices have the following form:

$$
M^{\ell} \sim\left\langle\phi_{d}\right\rangle\left(\begin{array}{ccc}
\lambda_{2}^{4} & 0 & \lambda_{1}^{2} \lambda_{2}^{3} \\
\lambda_{1}^{2} \lambda_{2}^{2} & \lambda_{1} \lambda_{2}^{2} & \lambda_{1}^{4} \lambda_{2} \\
0 & 0 & \lambda_{1} \lambda_{2}
\end{array}\right), \quad M^{\nu} \sim \frac{\left\langle\phi_{u}\right\rangle^{2}}{\tilde{M}}\left(\begin{array}{ccc}
\lambda_{1}^{2} \lambda_{2}^{4} & \lambda_{1}^{4} \lambda_{2}^{2} & \lambda_{1} \lambda_{2}^{2} \\
\lambda_{1}^{4} \lambda_{2}^{2} & \lambda_{1}^{6} & \lambda_{1}^{3} \\
\lambda_{1} \lambda_{2}^{2} & \lambda_{1}^{3} & 1
\end{array}\right)
$$

It predicts:

$$
\sin \theta_{23} \sim \lambda^{3}, \quad \sin \theta_{13} \sim \lambda^{5}, \quad m_{\nu_{e}} / m_{\nu_{\mu}} \sim \lambda^{4}
$$

In this example, the relations (3.2) and (3.7) are maintained, but (3.3) and (3.5) are circumvented due to the holomorphy of the superpotential.

\section{Exact Relations}

We investigate the possibility that the horizontal symmetry dictates not just order of magnitude relations but also exact (typically to order $\lambda$ or $\lambda^{2}$ ) relations. That can be the case if there are enough zeros in the mass matrices. These zeros arise from the holomorphy of the superpotential, when a certain entry breaks $\mathcal{H}$ by a negative charge.

Exact predictions arise trivially in the case that $m_{\nu_{\mu}} / m_{\nu_{\tau}} \sim \lambda^{5}$ as will be discussed in section 9. More generally, if any ratio between neutrino masses is an odd power of the small breaking parameter, then the mixing angle between the two neutrinos will vanish. This is a result of a symmetry and therefore exact to all orders.

We now investigate the possibility of exact relations in the case $m_{\nu_{\mu}} / m_{\nu_{\tau}} \sim \lambda^{4}$, described in section 4.1. We used the techniques for diagonalizing the mass matrices described in refs. [13] [5]. Let us recall that the mixing angles are given by $\left(s_{i j} \equiv \sin \theta_{i j}\right)$

$$
\begin{aligned}
& s_{12}=\left|s_{12}^{\ell}-s_{12}^{\nu}\right|, \\
& s_{23}=\left|s_{23}^{\ell}-s_{23}^{\nu}\right|, \\
& s_{13}=\left|s_{13}^{\ell}-s_{13}^{\nu}-s_{12}^{\nu}\left(s_{23}^{\ell}-s_{23}^{\nu}\right)\right|,
\end{aligned}
$$


where $s_{i j}^{\nu}\left(s_{i j}^{\ell}\right)$ are the elements of $V_{L}^{\nu}\left(V_{L}^{\ell}\right)$ where

$$
\begin{aligned}
V_{L}^{\nu} M^{\nu}\left(V_{L}^{\nu}\right)^{T} & =\operatorname{diag}\left(m_{\nu_{e}}, m_{\nu_{\mu}}, m_{\nu_{\tau}}\right) \\
V_{L}^{\ell} M^{\ell}\left(V_{R}^{\ell}\right)^{\dagger} & =\operatorname{diag}\left(m_{e}, m_{\mu}, m_{\tau}\right)
\end{aligned}
$$

For the neutrinos, we define

$$
\begin{aligned}
& y_{i j}^{\nu} \equiv M_{i j}^{\nu} / M_{33}^{\nu}, \\
& \tilde{y}_{22}^{\nu}=y_{22}^{\nu}-y_{23}^{\nu} y_{32}^{\nu} .
\end{aligned}
$$

(Note: $y_{33}^{\nu}=1 ;\left|\tilde{y}_{22}^{\nu}\right|=m_{\nu_{\mu}} / m_{\nu_{\tau}}$.) The $s_{i j}^{\nu}$ mixing angles are (we write only the potentially leading terms for $M^{\nu}$ of (4.6))

$$
s_{12}^{\nu}=\frac{y_{12}^{\nu}}{\tilde{y}_{22}^{\nu}}-\frac{y_{13}^{\nu} y_{32}^{\nu}}{\tilde{y}_{22}^{\nu}}, \quad s_{13}^{\nu}=y_{13}^{\nu}, \quad s_{23}^{\nu}=y_{23}^{\nu}
$$

For the charged leptons, we define

$$
\begin{aligned}
y_{i 1}^{\ell} & =\frac{M_{i 1}^{\ell}}{\sqrt{\left|M_{32}^{\ell}\right|^{2}+\left|M_{33}^{\ell}\right|^{2}}}, \\
y_{i 2}^{\ell} & =\frac{M_{i 2}^{\ell} M_{33}^{\ell}-M_{i 3}^{\ell} M_{32}^{\ell}}{\left|M_{32}^{\ell}\right|^{2}+\left|M_{33}^{\ell}\right|^{2}}, \\
y_{i 3}^{\ell} & =\frac{M_{i 3}^{\ell} M_{33}^{\ell}-M_{i 2}^{\ell} M_{32}^{\ell}}{\left|M_{32}^{\ell}\right|^{2}+\left|M_{33}^{\ell}\right|^{2}} .
\end{aligned}
$$

The $s_{i j}^{\ell}$ mixing angles are (we write only the potentially leading terms for $M^{\ell}$ of (4.6))

$$
s_{12}^{\ell}=\frac{y_{12}^{\ell}}{y_{22}^{\ell}}, \quad s_{13}^{\ell}=y_{13}^{\ell}, \quad s_{23}^{\ell}=y_{23}^{\ell} .
$$

We find two possible exact relations. First, take mass matrices of the form

$$
M^{\ell} \sim\left\langle\phi_{d}\right\rangle\left(\begin{array}{ccc}
\lambda^{8} & 0 & \lambda^{7} \\
\lambda^{6} & \lambda^{5} & \lambda^{5} \\
\lambda^{4} & 0 & \lambda^{3}
\end{array}\right), \quad M^{\nu} \sim \frac{\left\langle\phi_{u}\right\rangle^{2}}{M_{L}}\left(\begin{array}{ccc}
0 & \lambda^{6} & 0 \\
\lambda^{6} & \lambda^{4} & \lambda^{2} \\
0 & \lambda^{2} & 1
\end{array}\right)
$$

With $M_{13}^{\nu}=0$ we have $s_{13}^{\nu}=0$, while with $M_{12}^{\ell}=M_{32}^{\ell}=0$ we have $s_{12}^{\ell}=0$. Then

$$
\frac{m_{\nu_{e}}}{m_{\nu_{\mu}}}=s_{12}^{2}
$$


An example of charges that lead to (5.7) is a symmetry of the type (4.10) with

$\begin{array}{cccccccc}L_{1} & L_{2} & L_{3} & \bar{\ell}_{1} & \bar{\ell}_{2} & \bar{\ell}_{3} & \phi_{u} & \phi_{d} \\ (6,-1) & (0,1) & (0,0) & (2,1) & (5,-1) & (1,1) & (0,0) & (0,0) .\end{array}$

Second, take mass matrices of the form

$$
M^{\ell} \sim\left\langle\phi_{d}\right\rangle\left(\begin{array}{ccc}
\lambda^{8} & \lambda^{7} & \lambda^{7} \\
\lambda^{6} & \lambda^{5} & 0 \\
\lambda^{4} & 0 & \lambda^{3}
\end{array}\right), \quad M^{\nu} \sim \frac{\left\langle\phi_{u}\right\rangle^{2}}{M_{L}}\left(\begin{array}{ccc}
\lambda^{10} & \lambda^{8} & \lambda^{6} \\
\lambda^{8} & 0 & \lambda^{4} \\
\lambda^{6} & \lambda^{4} & \lambda^{2}
\end{array}\right) .
$$

With $M_{22}^{\nu}=0$ we have $m_{\nu_{\mu}} / m_{\nu_{\tau}}=\left(s_{23}^{\nu}\right)^{2}$, while with $M_{23}^{\ell}=M_{32}^{\ell}=0$ we have $s_{23}^{\ell}=0$. Then

$$
\frac{m_{\nu_{\mu}}}{m_{\nu_{\tau}}}=s_{23}^{2} .
$$

An example of charges that lead to (5.10) is a symmetry of the type (4.10) with

$$
\begin{array}{cccccccc}
L_{1} & L_{2} & L_{3} & \bar{\ell}_{1} & \bar{\ell}_{2} & \bar{\ell}_{3} & \phi_{u} & \phi_{d} \\
(1,2) & (-1,2) & (1,0) & (1,1) & (4,-1) & (0,1) & (0,0) & (0,0) .
\end{array}
$$

To summarize, the generic order of magnitude relations between neutrino mass ratios and mixing angles (3.2) may be promoted to exact relations (for $m_{\nu_{e}} / m_{\nu_{\mu}}$ or $m_{\nu_{\mu}} / m_{\nu_{\tau}}$ ) if holomorphy induces certain zero entries in the lepton mass matrices.

\section{A Full High Energy Theory}

So far we have considered a low energy effective theory with an explicitly broken horizontal symmetry $\mathcal{H}$ that gives certain selection rules. The most natural full high energy theory where our model can be embedded is that of Froggatt and Nielsen [14]. $\mathcal{H}$ is an exact symmetry that is spontaneously broken by a vev $\langle S\rangle$ of a scalar field that is a singlet under the SM gauge group. The information about the breaking is communicated to the observed leptons by heavy leptons in vector representations. The mass scale for these lepton supermultiplets is denoted by $M$, and the small parameter $\lambda=\langle S\rangle / M$. The relevant $S U(2)_{L} \times U(1)_{Y} \times U(1)_{H}$ representations of the heavy leptons are

$$
\begin{gathered}
F(2,-1 / 2, H) \text { and } \bar{F}(2,+1 / 2,-H), \\
E(1,-1, H) \text { and } \bar{E}(1,+1,-H), \\
N(1,0, H) \text { and } \bar{N}(1,0,-H) .
\end{gathered}
$$


An important question is whether any of these new particles can be observed in experiment, thus providing direct evidence for the horizontal symmetry framework. The answer depends on the scales $\langle S\rangle$ and $M$ : the low energy parameters determine only the ratio between them. If they are low enough, say $M \lesssim 500 \mathrm{TeV}$, then the particles may, in principle, be accessible in future experiments. Lower bounds on these scales come from flavor changing neutral currents (FCNC) and from requiring that no Landau poles appear in the running of the gauge couplings below the Planck scale [3] [5].

The scalar $S$ mediates various lepton flavor violating processes, e.g. $\mu \rightarrow$ eee and $K \rightarrow \pi \mu e$. We assume that the mass of $S$ is of the order of the SUSY breaking scale (see the analysis of the Higgs potential in ref. [5]), and that its coupling to a fermion pair $f_{i} \bar{f}_{j}$ is of order $\frac{\left(m_{i}+m_{j}\right) \sin \theta_{i j}}{\langle S\rangle}$. Surveying all relevant FCNC processes (for a recent review, see [15]), we find that all the bounds on $\langle S\rangle$ are below the electroweak scale. Similarly, the new leptons contribute to lepton flavor violating processes through loop diagrams, but no significant bounds arise.

The $N, \bar{N}$ supermultiplets do not affect the running of the gauge couplings. The number of $F$-doublets $N_{F}\left(=N_{\bar{F}}\right)$ and the number of $E$-singlets $N_{E}\left(=N_{\bar{E}}\right)$ affect the running of the $S U(2)_{L}$ and $U(1)_{Y}$ coupling constants and may lead to Landau poles. The relevant counting is [5]

$$
\begin{aligned}
& N_{2}=3 N_{P}+N_{F}, \\
& N_{1}=N_{P}+8 N_{U}+2 N_{D}+3 N_{F}+6 N_{E},
\end{aligned}
$$

where $N_{P}$ is the number of quark doublets and $N_{U}\left(N_{D}\right)$ is the number of quark singlets of charge $+2 / 3(-1 / 3)$. If, for example, we are interested in $M \leq 400 \mathrm{TeV}$, then $N_{2} \leq 5$ and $N_{1} \leq 18$.

The minimal number of heavy leptons required can be easily deduced from the suppression of the light masses. For the model defined by (4.5), Eq. (4.6) gives $\operatorname{det} M^{\ell} \sim\left\langle\phi_{d}\right\rangle^{3} \lambda^{16}$, which requires $N_{F}+N_{E} \geq 16$, (if $m_{\tau} /\left\langle\phi_{d}\right\rangle \sim 1$, $\operatorname{det} M^{\ell} \sim\left\langle\phi_{d}\right\rangle^{3} \lambda^{7}$ and $N_{F}+N_{E} \geq 7$ ), and $\operatorname{det} M^{\nu} \sim \frac{\left\langle\phi_{u}\right\rangle^{6}}{M^{3}} \lambda^{12}$, which requires $N_{F}+N_{N} \geq 12$ and $N_{\hat{N}}=3$ where $\hat{N}$ are $\mathcal{H}$ singlets $(\hat{N}(1,0,0))$. We will assume that all heavy neutrinos are $S U(2)_{L}$ singlets, in which case they are irrelevant to the investigation of Landau poles. There is no way to 
have $N_{F}+N_{E} \geq 7, N_{F} \leq N_{2} \leq 5$ and $3 N_{F}+6 N_{E} \leq N_{1} \leq 18$. Therefore, if the smallness of lepton masses is a result of a horizontal $U(1)_{H}$ symmetry, the breaking of the symmetry is at an energy too high for direct observation.

The lowest possible scale in our framework is allowed when $H=U(1)_{H_{1}} \times U(1)_{H_{2}}$, $\lambda_{1} \sim \lambda^{2}$ and $\lambda_{2} \sim \lambda^{3}$. Here we could imagine $m_{\mu} / m_{\tau} \sim \lambda_{1}, m_{e} / m_{\tau} \sim \lambda_{1} \lambda_{2}$ and $m_{\tau} /\left\langle\phi_{d}\right\rangle \sim$ 1. In such a case, only 3 heavy charged leptons are needed, so we can have $N_{F}=2$ and $N_{E}=1$. As shown in ref. [5], this - together with a specific choice of quark representations - allows $M \geq 900 \mathrm{TeV}$.

To summarize, a natural embedding of our low energy effective theory in a full high energy framework requires the existence of scalars with flavor changing couplings and new leptons in vector representations. Constraints on the masses of these new particles from FCNC are very mild. However, if the gauge group is that of the Standard Model up to the Planck scale, then Landau poles constraints make it very unlikely that the new particles are light enough to be directly observed in future experiments.

We would like to emphasize the following point [16]: Our various predictions for the light neutrino parameters, e.g. the predictions in section 3, are independent of the structure of the right-handed neutrino mass matrix. This is in contrast to most models of see-saw mass matrices where one has to assume a specific hierarchy in the mass matrix for the heavy neutrinos in order to have any prediction for the light spectrum. The point can be proved as follows. Denote by $M_{R}$ the Majorana mass matrix for the heavy $N$ fields, and by $M_{D}$ the Dirac mass matrix. Then

$$
\left[M^{\nu}\right]_{i j} \sim\left[M_{D}\right]_{i k}\left[\left(M_{R}\right)^{-1}\right]_{k l}\left[M_{D}^{T}\right]_{l j}
$$

Assume that $N_{k}$ carries horizontal charge $H_{k}$. Then $\left[M_{D}\right]_{i k} \propto \lambda^{H_{i}+H_{k}},\left[M_{D}\right]_{j l} \propto \lambda^{H_{j}+H_{l}}$, and $\left[M_{R}\right]_{k l} \propto \lambda^{H_{k}+H_{l}}$. Our selection rules further imply that $\left[\left(M_{R}\right)^{-1}\right]_{k l} \sim\left(\left[M_{R}\right]_{k l}\right)^{-1}$. Then the product $\left[M_{D}\right]_{i k}\left[\left(M_{R}\right)^{-1}\right]_{k l}\left[M_{D}^{T}\right]_{l j}$ is independent of both $H_{k}$ and $H_{l}$. Our low energy predictions are valid for arbitrary structure of $M_{R}$ (as long as it has no zero eigenvalues). 


\section{Lepton Slepton Alignment}

For generic slepton masses, penguin diagrams with sleptons and neutralinos give unacceptably large contributions to radiative charged lepton decays [17]. The standard solution to this problem is to assume that sleptons are degenerate to a very good approximation. This is not motivated in generic supergravity models or string theory, though it may hold under special conditions [18]. Both slepton degeneracy and proportionality of trilinear Higgs-slepton couplings to Yukawa couplings can be natural if Supersymmetry breaking is communicated to the light particles by gauge interactions [19], or in models with a non-Abelian horizontal symmetry [20].

Recently, an alternative mechanism has been suggested to solve the FCNC problem in the quark sector [4] [5]: the approximate alignment of quark mass matrices with squark mass-squared matrices. In this section we investigate whether such a mechanism can work for the lepton sector as well. The idea is that a horizontal symmetry, of the type discussed in this work, forces both $M^{f}$ and $\tilde{M}^{f 2}(f=\ell, \nu)$ to be approximately diagonal in the basis where the horizontal charges are well defined. Consequently, the mixing matrix for lepton-slepton-photino couplings is close to a unit matrix and FCNCs are suppressed, regardless of whether sleptons are degenerate or not.

To explicitly investigate this mechanism, we define diagonalizing matrices $V_{M}^{f}$ and $\tilde{V}_{M}^{f}$ :

$$
\begin{gathered}
V_{L}^{\ell} M^{\ell} V_{R}^{\ell \dagger}=\operatorname{diag}\left\{m_{e}, m_{\mu}, m_{\tau}\right\}, \\
V_{L}^{\nu} M^{\nu} V_{L}^{\nu T}=\operatorname{diag}\left\{m_{\nu_{e}}, m_{\nu_{\mu}}, m_{\nu_{\tau}}\right\}, \\
\tilde{V}_{L}^{f} \tilde{M}_{L L}^{f 2} \tilde{V}_{L}^{f \dagger}=\operatorname{diag}\left\{m_{\tilde{f}_{L 1}}^{2}, m_{\tilde{f}_{L 2}}^{2}, m_{\tilde{f}_{L 3}}^{2}\right\}, \\
\tilde{V}_{R}^{\ell} \tilde{M}_{R R}^{\ell 2} \tilde{V}_{R}^{\ell \dagger}=\operatorname{diag}\left\{m_{\tilde{e}_{R}}^{2}, m_{\tilde{\mu}_{R}}^{2}, m_{\tilde{\tau}_{R}}^{2}\right\} .
\end{gathered}
$$

We assume here that the average slepton mass, $m_{\tilde{\ell}}$ and $m_{\tilde{\nu}}$, is somewhat higher than the electroweak breaking scale $m_{Z}$, so that $\tilde{M}_{L L}^{\ell 2} \approx \tilde{M}_{L L}^{\nu 2}$. The neutralino mixing matrices are then

$$
K_{L}^{f}=V_{L}^{f} \tilde{V}_{L}^{f \dagger}, \quad K_{R}^{\ell}=V_{R}^{\ell} \tilde{V}_{R}^{\ell \dagger}
$$

Supersymmetric contributions to FCNC processes will be proportional to

$$
\left(\delta_{M M}^{f}\right)_{i j} \sim \max _{\alpha}\left(K_{M}^{f}\right)_{i \alpha}\left(K_{M}^{f}\right)_{j \alpha}^{*}
$$


Furthermore, the off-diagonal blocks are suppressed by $\mathcal{O}\left(m_{f} / m_{\tilde{f}}\right)$ compared to the diagonal blocks. Their contributions to FCNC are then proportional to

$$
\left(\delta_{L R}^{\ell}\right)_{i j}=\left(V_{L}^{\ell} \tilde{M}_{L R}^{\ell 2} V_{R}^{\ell \dagger}\right)_{i j} / m_{\tilde{\ell}}^{2}
$$

We have checked the bounds on $\left(\delta_{M N}^{f}\right)_{i j}$ from all relevant lepton flavor changing processes. The strongest bounds come from radiative lepton decays. We find [17] (using $\left.m_{\tilde{\gamma}}^{2} / m_{\tilde{\ell}}^{2}=0.5\right)$ :

$$
\begin{gathered}
B R(\mu \rightarrow e \gamma) \leq 4.9 \times 10^{-11} \Longrightarrow\left\{\begin{array}{l}
\left(\delta_{M M}^{\ell}\right)_{12} \leq 1 \times 10^{-1}\left(\frac{m_{\tilde{\ell}}}{1 T_{\tilde{\ell} V}}\right)^{2}, \\
\left(\delta_{L R}^{\ell}\right)_{12} \leq 2 \times 10^{-5}\left(\frac{m_{\tilde{\ell}}}{1 T e V}\right) .
\end{array}\right. \\
B R(\tau \rightarrow e \gamma) \leq 2.0 \times 10^{-4} \Longrightarrow\left\{\begin{array}{l}
\left(\delta_{M M}^{\ell}\right)_{13} \leq 4 \times 10^{3}\left(\frac{m_{\tilde{e}}}{1 T e V}\right)^{2}, \\
\left(\delta_{L R}^{\ell}\right)_{13} \leq 2\left(\frac{m_{\tilde{\ell}}}{1 T e V}\right) .
\end{array}\right. \\
B R(\tau \rightarrow \mu \gamma) \leq 4.2 \times 10^{-6} \Longrightarrow\left\{\begin{array}{l}
\left(\delta_{M M}^{\ell}\right)_{23} \leq 7 \times 10^{2}\left(\frac{m_{\tilde{\ell}}}{1 T e_{\tilde{\ell}}}\right)^{2} \\
\left(\delta_{L R}^{\ell}\right)_{23} \leq 2 \times 10^{-1}\left(\frac{m_{\tilde{\ell}}}{1 T e V}\right) .
\end{array}\right.
\end{gathered}
$$

For the $\delta_{L R}^{\ell}$ matrix, the horizontal symmetry implies

$$
\left(\tilde{M}_{L R}^{\ell 2}\right)_{i j} \sim m_{\tilde{\ell}} M_{i j}^{\ell}
$$

which gives, with the choice (4.5),

$$
\begin{aligned}
& \left(\delta_{L R}^{\ell}\right)_{12} \sim \frac{m_{\mu} \sin \theta_{12}}{m_{\tilde{\ell}}} \sim 4 \times 10^{-6}\left(\frac{1 T e V}{m_{\tilde{\ell}}}\right), \\
& \left(\delta_{L R}^{\ell}\right)_{13} \sim \frac{m_{\tau} \sin \theta_{13}}{m_{\tilde{\ell}}} \sim 3 \times 10^{-6}\left(\frac{1 T e V}{m_{\tilde{\ell}}}\right), \\
& \left(\delta_{L R}^{\ell}\right)_{23} \sim \frac{m_{\tau} \sin \theta_{23}}{m_{\tilde{\ell}}} \sim 7 \times 10^{-5}\left(\frac{1 T e V}{m_{\tilde{\ell}}}\right) .
\end{aligned}
$$

We see that the constraints on $\left(\delta_{L R}^{\ell}\right)_{i j}$ are satisfied. (For $\mu \rightarrow e \gamma$, (7.6) and (7.10) imply $m_{\tilde{\ell}} \geq 200 \mathrm{GeV}$.)

For the $\delta_{M M}^{\ell}$ matrices, the $\left(\delta_{M M}^{\ell}\right)_{i 3}$ elements are not really constrained. On the other hand,

$$
\left(\delta_{M M}^{\ell}\right)_{12} \lesssim \lambda^{2}
$$


is required. (For $m_{\tilde{\ell}} \gtrsim 1 \mathrm{TeV}$ the bound is relaxed to $\lambda$.) As we require $\sin \theta_{12} \sim \lambda^{2}$ and $m_{e} / m_{\mu} \sim \lambda^{3}$, the naive estimates are

$$
\left(\delta_{L L}^{\ell}\right)_{12} \lesssim \lambda^{2}, \quad\left(\delta_{R R}^{\ell}\right)_{12} \lesssim \lambda .
$$

The only potential problem is then with $\left(\delta_{R R}^{\ell}\right)_{12}$, and even this is automatically solved if $m_{\tilde{\ell}}$ is somewhat on the heavy side. Below we present an example of a horizontal symmetry that aligns leptons and sleptons of the first two generations so that $\left(K_{R}^{\ell}\right)_{12}$ is satisfactorily suppressed even for light sleptons.

Take $\mathcal{H}=U(1)_{H_{1}} \times U(1)_{H_{2}}, \lambda_{1} \sim \lambda$ and $\lambda_{2} \sim \lambda^{2}$, and charge assignments

$\begin{array}{cccccccc}L_{1} & L_{2} & L_{3} & \bar{\ell}_{1} & \bar{\ell}_{2} & \bar{\ell}_{3} & \phi_{u} & \phi_{d} \\ (0,2) & (2,0) & (0,0) & (6,-1) & (1,1) & (1,1) & (0,0) & (0,0) .\end{array}$

It leads to the following mass matrices for charged leptons and sleptons:

$$
\begin{gathered}
M^{\ell} \sim\left\langle\phi_{d}\right\rangle\left(\begin{array}{ccc}
\lambda_{1}^{6} \lambda_{2} & \lambda_{1} \lambda_{2}^{3} & \lambda_{1} \lambda_{2}^{3} \\
0 & \lambda_{1}^{3} \lambda_{2} & \lambda_{1}^{3} \lambda_{2} \\
0 & \lambda_{1} \lambda_{2} & \lambda_{1} \lambda_{2}
\end{array}\right), \\
\tilde{M}_{L L}^{\ell 2} \sim m_{\tilde{\ell}}^{2}\left(\begin{array}{ccc}
1 & \lambda_{1}^{2} \lambda_{2}^{2} & \lambda_{2}^{2} \\
\lambda_{1}^{2} \lambda_{2}^{2} & 1 & \lambda_{1}^{2} \\
\lambda_{2}^{2} & \lambda_{1}^{2} & 1
\end{array}\right), \tilde{M}_{R R}^{\ell 2} \sim m_{\tilde{\ell}}^{2}\left(\begin{array}{ccc}
1 & \lambda_{1}^{5} \lambda_{2}^{2} & \lambda_{1}^{5} \lambda_{2}^{2} \\
\lambda_{1}^{5} \lambda_{2}^{2} & 1 & 1 \\
\lambda_{2}^{5} \lambda_{2}^{2} & 1 & 1
\end{array}\right) .
\end{gathered}
$$

In this model

$$
\begin{aligned}
& \left(V_{L}^{\ell}\right)_{12} \sim \lambda^{2}, \quad\left(\tilde{V}_{L}^{\ell}\right)_{12} \sim \lambda^{6} \Longrightarrow\left(\delta_{L L}^{\ell}\right)_{12} \sim \lambda^{2}, \\
& \left(V_{R}^{\ell}\right)_{12} \sim \lambda^{5}, \quad\left(\tilde{V}_{R}^{\ell}\right)_{12} \sim \lambda^{9} \Longrightarrow\left(\delta_{R R}^{\ell}\right)_{12} \sim \lambda^{5},
\end{aligned}
$$

and all constraints are satisfied.

To summarize, the horizontal symmetry aligns lepton mass matrices with slepton mass-squared matrices. If slepton masses are of $\mathcal{O}(1 \mathrm{TeV})$, then SUSY penguin diagrams contributions to radiative lepton decays are below the experimental bounds and no slepton degeneracy needs to be assumed. If sleptons are lighter than $\mathcal{O}(1 \mathrm{TeV})$, then either $\frac{m_{\tilde{e}_{R}}^{2}-m_{\tilde{\mu}_{R}}^{2}}{m_{\tilde{\ell}_{R}}^{2}} \lesssim 0.2$, or the combination of horizontal symmetry and holomorphy produces an alignment that is more precise than the naive estimate.

\section{Naturally Light Leptoquarks}

Light $\left(M_{L Q}=\mathcal{O}(T e V)\right)$ leptoquarks may be discovered in the DESY ep collider HERA and in Fermilab. However, light leptoquarks pose severe phenomenological problems unless 
their couplings are chiral and diagonal (see e.g. ref. [21]). Horizontal symmetries are necessary to guarantee these features in a natural way [6]. In the analysis of ref. [6], it was assumed that neutrinos are massless. In this section we extend their analysis to the case of massive neutrinos. Also, while ref. [6] presented a single example, we show that actually a rather large class of models allows light leptoquarks.

Following ref. [6], we focus on the case motivated by $E_{6}$ (or superstring) models, where the leptoquark supermultiplets are in the following $S U(3)_{C} \times S U(2)_{L} \times U(1)_{Y}$ representations:

$$
S(\overline{3}, 1)_{+1 / 3}, \quad S^{\prime}(3,1)_{-1 / 3} .
$$

In the superpotential, the following renormalizable couplings appear:

$$
\left(G_{L}\right)_{i j} L_{i} Q_{j} S+\left(G_{R}\right)_{i j} \bar{\ell}_{i} \bar{u}_{j} S^{\prime}
$$

(where $Q(3,2)_{+1 / 6}$ and $\bar{u}(\overline{3}, 1)_{-2 / 3}$ are quark supermultiplets). The phenomenologically relevant quantities are the coupling matrices in the mass basis,

$$
G_{L}^{\nu d}=V^{\nu \dagger} G_{L} V_{L}^{d}, \quad G_{L}^{\ell u}=V_{L}^{\ell \dagger} G_{L} V_{L}^{u}, \quad G_{R}^{\ell u}=V_{R}^{\ell \dagger} G_{R} V_{R}^{u}
$$

(The matrices $V_{L, R}^{d, u}$ are defined analogously to the lepton diagonalizing matrices, see Eq. $(7.1)$.

We are interested in finding models where the $S$ leptoquark is allowed to have a light mass while its diagonal coupling to first generation fermions is not suppressed, namely

$$
M_{S}=\mathcal{O}(T e V), \quad\left(G_{L}^{\nu d}\right)_{11}=\mathcal{O}(1), \quad\left(G_{L}^{\ell u}\right)_{11}=\mathcal{O}(1)
$$

This task is difficult because off-diagonal couplings have to be highly suppressed [6] [21] [22]. In particular, $\mu-e$ conversion in nuclei requires

$$
\left(G_{L}^{\ell u}\right)_{21} \leq 10^{-4}\left(\frac{M_{S}}{1 T e V}\right)
$$

while $K-\bar{K}$ mixing requires

$$
\left(G_{L}^{\nu d}\right)_{12} \leq 3 \times 10^{-2}\left(\frac{M_{S}}{1 T e V}\right)
$$


Finally, we need to allow a mixing term in the superpotential for the $S$ and $S^{\prime}$ field, $\mu_{S S^{\prime}} S S^{\prime}$, to give masses to the fermionic components, but then the $G_{R}$ matrix has to be highly suppressed to avoid inconsistency with the measured leptonic decays of the pion:

$$
\mu_{S S^{\prime}} \gtrsim m_{Z}, \quad\left(G_{R}^{\ell u}\right)_{i j} \ll 1 .
$$

A horizontal symmetry is essential in naturally fulfilling these requirements in three important ways:

a. In the interaction basis, $\mathcal{H}$ can give

$$
G_{L} \sim\left(\begin{array}{ccc}
1 & 0 & 0 \\
0 & 0 & 0 \\
0 & 0 & 0
\end{array}\right), \quad G_{R} \sim\left(\begin{array}{ccc}
0 & 0 & 0 \\
0 & 0 & 0 \\
0 & 0 & 0
\end{array}\right),
$$

where by zero entry we mean that it is highly suppressed $\left(\sim \lambda^{n}\right.$ with $\left.n \gg 1\right)$.

b. In the mass basis, the off diagonal couplings are still small because the horizontal symmetry forces the lepton mass matrices and quark mass matrices to be simultaneously approximately diagonal.

c. The horizontal symmetry together with holomorphy can make specific couplings particularly small (similar to the quark squark alignment where gluino couplings in the down sector are highly suppressed).

We now explain these points in more detail.

$a$. In order to make $\left(G_{L}\right)_{11}$ the only unsuppressed leptoquark coupling, we can choose

$$
H(S)+H\left(Q_{1}\right)+H\left(L_{1}\right)=0 ; \quad H\left(S^{\prime}\right)=-H(S) .
$$

As $H\left(Q_{i}\right)+H\left(L_{j}\right)<H\left(Q_{1}\right)+H\left(L_{1}\right)$ for $i$ and/or $j \neq 1,\left(G_{L}\right)_{i j}$ would either vanish (due to holomorphy) or be highly suppressed if $\mathcal{H}$ is discrete. Also $\left(G_{R}\right)_{i j} \sim$ $\lambda^{H\left(Q_{1}\right)+H\left(L_{1}\right)+H\left(\bar{\ell}_{i}\right)+H\left(\bar{u}_{j}\right)}$ would be highly suppressed. Effectively, we get (8.8).

Three comments are in order:

(i) The term $\mu_{S S^{\prime}} S S^{\prime}$ in the superpotential, which is necessary to give the fermionic components of $S$ and $S^{\prime}$ masses, is allowed.

(ii) The $\left(G_{R}\right)_{i j}$ couplings can be further suppressed by choosing horizontal charges $\tilde{H}=H+\alpha X$ where $X\left(\phi_{d}\right)=-X\left(\bar{d}_{i}\right)=-X\left(\bar{\ell}_{i}\right)$ and all other fields carry $X=0 . U(1)_{X}$ is 
an accidental symmetry of the Yukawa matrices. Consequently, $H$ and $\tilde{H}$ are isomorphic as far as fermion masses and mixings are concerned.

(iii) Another way to suppress the $G_{R}$ matrix is by taking $H\left(S^{\prime}\right)>-H(S)$. In this way also $\mu_{S S^{\prime}}$ is suppressed, but its scale is anyway unknown.

$b$. The horizontal symmetry guarantees that the various elements in the diagonalizing matrices are not larger than the corresponding elements in the mixing matrices, e.g. $\left(V_{L}^{u}\right)_{12},\left(V_{L}^{d}\right)_{12} \lesssim V_{u s}$. ( $V$ here is the CKM mixing matrix for quarks. Below we denote the charged current mixing matrix for leptons by $U$.) Consequently,

$$
\begin{aligned}
& \left(G_{L}^{\ell u}\right)_{i j} \sim\left(V_{L}^{\ell}\right)_{1 i}\left(V_{L}^{u}\right)_{1 j} \lesssim U_{1 i} V_{1 j} \\
& \left(G_{L}^{\nu d}\right)_{i j} \sim\left(V_{L}^{\nu}\right)_{1 i}\left(V_{L}^{d}\right)_{1 j} \lesssim U_{1 i} V_{1 j}
\end{aligned}
$$

Thus, for example, as $V_{12} \sim \lambda$ and we assume $U_{12} \sim \lambda^{2}$, the bound on $M_{S}$ from (8.5) relaxes by a factor of $\lambda^{2}$ to $M_{S} \gtrsim 400 \mathrm{TeV}$, while the bound (8.6) relaxes by a factor of $\lambda$ to $M_{S} \gtrsim 8 \mathrm{TeV}$.

c. The strongest bounds come from the charged lepton and down sectors. Thus, the lower bounds on $M_{S}$ would be weakest in models where

$$
\left(V_{L}^{d}\right)_{12} \ll V_{12} \sim \lambda, \quad\left(V_{L}^{\ell}\right)_{12} \ll U_{12} \sim \lambda^{2} .
$$

These are precisely the models of quark-squark alignment (see ref. [5]) and lepton-slepton alignment (see the previous section). By using quark squark alignment, the bound from $K-\bar{K}$ mixing is avoided, and only a weaker bound from $D-\bar{D}$ mixing holds:

$$
M_{S} \geq \mathcal{O}(5 T e V)
$$

By using lepton slepton alignment, the bound from electron muon conversion can be relaxed to below $\mathrm{TeV}$. Note that the unavoidable bound from lepton universality in pion decay, $M_{S} \gtrsim 3.4 \mathrm{TeV}$, holds in all models [21].

We now present an explicit example. Let the horizontal symmetry group be $\mathcal{H}=$ $U(1)_{H_{1}} \times U(1)_{H_{2}}$, with breaking parameters $\lambda_{1} \sim \lambda$ and $\lambda_{2} \sim \lambda^{2}$. Assume the following 
charge assignments:

$\begin{array}{cccccccc}L_{1} & L_{2} & L_{3} & \bar{\ell}_{1} & \bar{\ell}_{2} & \bar{\ell}_{3} & \phi_{u} & \phi_{d} \\ (0,2) & (2,0) & (0,0) & (6,-1) & (-1,2) & (1,1) & (0,0) & (0,0) \\ Q_{1} & Q_{2} & Q_{3} & \bar{d}_{1} & \bar{d}_{2} & \bar{d}_{3} & S & S^{\prime} \\ (3,0) & (0,1) & (0,0) & (-1,2) & (4,-1) & (0,1) & (-3,-2) & (3,2) \\ & & & \bar{u}_{1} & \bar{u}_{2} & \bar{u}_{3} & & \\ & & & (-1,2) & (1,0) & (0,0) & & \end{array}$

It leads to the required hierarchy in quark and lepton parameters and to the following leptoquark couplings:

$$
G_{L}^{\ell u} \sim\left(\begin{array}{ccc}
1 & \lambda & \lambda^{3} \\
\lambda^{6} & \lambda^{7} & \lambda^{9} \\
\lambda^{4} & \lambda^{5} & \lambda^{7}
\end{array}\right), \quad G_{L}^{\nu d} \sim\left(\begin{array}{ccc}
1 & \lambda^{5} & \lambda^{3} \\
\lambda^{2} & \lambda^{7} & \lambda^{5} \\
\lambda^{4} & \lambda^{9} & \lambda^{7}
\end{array}\right) .
$$

Note in particular that $\left(G_{L}^{\ell u}\right)_{21} \sim \lambda^{6}$ so that (8.5) requires $M_{S} \gtrsim 600 \mathrm{GeV}$, while $\left(G_{L}^{\nu d}\right)_{12} \sim$ $\lambda^{5}$ so that (8.6) requires $M_{S} \gtrsim 10 \mathrm{GeV}$. In this model, the strongest bound comes from $D-\bar{D}$ mixing,

$$
M_{S} \gtrsim 20 \mathrm{TeV} \times\left(G_{L}^{\ell u}\right)_{12} \sim 5 \mathrm{TeV} .
$$

We conclude with two comments:

(i) The horizontal symmetry may allow, in similar ways to the models above, light leptoquarks that couple dominantly to second or third generation fermions. While these will not be directly produced in existing machines, they may affect precision measurements at the $Z^{0}$ pole [23].

(ii) The horizontal symmetry may allow light leptoquarks that couple dominantly to right-handed fermions, i.e. a light $S^{\prime}$. In this case, all bounds from FCNC can be avoided, and only the unavoidable bound from atomic parity violation [21], $M_{S^{\prime}} \gtrsim 2 \mathrm{TeV}$, holds.

\section{Exact Horizontal Symmetries}

If there is an exact horizontal symmetry in Nature, it implies either degeneracy between fermions or vanishing mixing angles [11][3]. In the quark sector, it is experimentally known that no two quarks are degenerate and that none of the CKM angles vanishes. Consequently, any horizontal symmetry acting non-trivially on quarks, has to be broken. However, as lepton mixing angles have not been experimentally determined, the possibility 
of an unbroken horizontal symmetry acting on the leptons remains open. In this section we investigate this possibility.

If we attribute to the breaking parameter $\lambda$ a unit $H$-charge, then a sub-group of the horizontal symmetry remains unbroken if there exist lepton fields with fractional $H$ charges. The selection rule (3.1) is modified into

$$
\sin \theta_{i j}=0 \text { for } H\left(L_{i}\right)-H\left(L_{j}\right)=\text { non }- \text { integer. }
$$

As an example, we take the case where $H\left(L_{1}\right)$ and $H\left(L_{2}\right)$ are half-integers while $H\left(L_{3}\right)$ is integer. Requiring that all charged lepton masses are non-vanishing, this would automatically require that $H\left(\bar{\ell}_{1}\right)$ and $H\left(\bar{\ell}_{2}\right)$ are half-integers while $H\left(\bar{\ell}_{3}\right)$ is integer. This can be easily modified to any other case. The three relations (3.2) are modified into

$$
\frac{m_{\nu_{e}}}{m_{\nu_{\mu}}} \sim \sin ^{2} \theta_{12}, \quad \sin \theta_{23}=\sin \theta_{13}=0
$$

Note that the last two predictions, $\sin \theta_{i 3}=0$, are not order of magnitude estimates but exact predictions: they result from an unbroken $Z_{2}$ symmetry, tau-parity $\left(R_{\tau}\right)$. Under $R_{\tau}$, $L_{3} \rightarrow-L_{3}, \bar{\ell}_{3} \rightarrow-\bar{\ell}_{3}$, while all other fields are $R_{\tau}$-even. Such symmetry has interesting phenomenological implications. It forbids $\Delta \tau=1$ processes such as $\tau \rightarrow \ell \gamma$ and $\tau \rightarrow \ell \ell \ell$ (where $\ell$ stands here for $e$ or $\mu$ ). In particular, $\nu_{\tau}-\nu_{\mu}$ and $\nu_{\tau}-\nu_{e}$ oscillations are forbidden, in which case there will be no signal in the CHORUS, NOMAD and E803 experiments. As $\nu_{\tau}$ is stable in this case, cosmological constraints imply that it should be lighter than $\mathcal{O}(100 \mathrm{eV})$.

Note that $R_{\tau}$ could be an exact symmetry even if we adopt the phenomenological input (1.3) and (1.4). The hierarchy in Eqs. (4.2) and (4.3) with

$$
m_{\nu_{\mu}} / m_{\nu_{\tau}} \sim \lambda^{5}
$$

determines a unique set of $H$ charge assignments:

$$
\begin{array}{cccccccc}
L_{1} & L_{2} & L_{3} & \bar{\ell}_{1} & \bar{\ell}_{2} & \bar{\ell}_{3} & \phi_{u} & \phi_{d} \\
(9 / 2) & (5 / 2) & (0) & (7 / 2) & (5 / 2) & (3) & (0) & (0) .
\end{array}
$$

The lepton mass matrices have then the following form:

$$
M^{\ell} \sim\left\langle\phi_{d}\right\rangle\left(\begin{array}{ccc}
\lambda^{8} & \lambda^{7} & 0 \\
\lambda^{6} & \lambda^{5} & 0 \\
0 & 0 & \lambda^{3}
\end{array}\right), \quad M^{\nu} \sim \frac{\left\langle\phi_{u}\right\rangle^{2}}{\tilde{M}}\left(\begin{array}{ccc}
\lambda^{9} & \lambda^{7} & 0 \\
\lambda^{7} & \lambda^{5} & 0 \\
0 & 0 & 1
\end{array}\right) .
$$


It predicts

$$
\sin \theta_{23}=0, \quad \sin \theta_{13}=0, \quad m_{\nu_{e}} / m_{\nu_{\mu}} \sim \lambda^{4}
$$

Both the form of the mass matrices (9.5) and the predicted values of the mixing angles (9.6) clearly show the consequences of tau-parity.

\section{Conclusions}

We have investigated the implications of supersymmetric Abelian horizontal symmetries on the lepton sector. We find a number of interesting predictions that hold in a large class of models:

(i) Mass ratios in the neutrino sector are of the order of the square of the corresponding mixing angles.

(ii) The mixing angles are larger than the corresponding ratios of charged lepton masses.

(iii) Mass ratios among neutrinos are larger than the square of the corresponding charged leptons mass ratios.

(iv) There is no inverted hierarchy among neutrinos.

The result (iii), when combined with cosmological constraints, implies that it is very likely that $m_{\nu_{e}}, m_{\nu_{\mu}}$ and $m_{\nu_{\tau}}$ are all lighter than $\mathcal{O}(100 \mathrm{eV})$ [12].

The embedding of the low energy selection rules in a full high energy theory, requires the existence of new particles. However, if the gauge symmetry does not change up to high scales, then these particles are very likely to be too heavy to be directly observed in experiments.

In addition to the various relations among lepton parameters, evidence for the horizontal symmetry framework may arise indirectly, from its implications on various other sectors of the theory. In particular, the horizontal symmetry suppresses SUSY contributions to lepton flavor violating processes to an acceptable level even if sleptons are not degenerate. If non-degenerate sleptons are discovered in experiment, then $\mu \rightarrow e \gamma$ is likely to be close to the experimental upper bound.

If light leptoquarks are discovered in experiment, then again the only likely mechanism to suppress their contributions to FCNC processes is the horizontal symmetry. $D-\bar{D}$ 
mixing is then likely to be close to the experimental upper bound.

acknowledgments

We thank Ernest Baver, Michael Dine, Enrico Nardi and Nati Seiberg for useful discus-

sions. YN is supported in part by the Israel Commission for Basic Research, by the United States - Israel Binational Science Foundation (BSF) and by the Minerva Foundation. 


\section{References}

[1] Particle Data Group, Phys. Rev. D50 (1994) 1173.

[2] For a recent analysis, see N. Hata and P. Langacker, Phys. Rev. D50 (1994) 632.

[3] M. Leurer, Y. Nir and N. Seiberg, Nucl. Phys. B398 (1993) 319.

[4] Y. Nir and N. Seiberg, Phys. Lett. B309 (1993) 337.

[5] M. Leurer, Y. Nir and N. Seiberg, Nucl. Phys. B420 (1994) 468.

[6] E. Baver and M. Leurer, Phys. Rev. D51 (1995) 260.

[7] H. Murayama and D.B. Kaplan, Phys. Lett. B336 (1994) 221.

[8] V. Ben-Hamo and Y. Nir, Phys. Lett. B339 (1994) 77.

[9] L. Ibáñez and G.G. Ross, Phys. Lett. B332 (1994) 100.

[10] H. Dreiner, G.K. Leontaris, S. Lola, G.G. Ross and C. Scheich, CERN/TH.7412/94, hep-ph/9409369.

[11] R. Gatto, G. Morchio and F. Strocchi, Phys. Lett. B83 (1979) 348;

R. Gatto, G. Morchio, G. Sartori and F. Strocchi, Nucl. Phys. B163 (1980) 221;

G. Segrè and H.A. Weldon, Phys. Lett. B86 (1979) 291; Ann. Phys. 124 (1980) 37.

[12] H. Harari and Y. Nir, Phys. Lett. B188 (1987) 163; Nucl. Phys. B292 (1987) 251.

[13] L.J. Hall and A. Rasin, Phys. Lett. B315 (1993) 164.

[14] C.D. Froggatt and H.B. Nielsen, Nucl. Phys. B147 (1979) 277.

[15] T.S. Kosmas, G.K. Leontaris and J.D. Vergados, Prog. Part. Nucl. Phys. 33 (1994) 397.

[16] A. Rasin and J.P. Silva, Phys. Rev. D49 (1994) 20.

[17] F. Gabbiani and A. Masiero, Nucl. Phys. B322 (1989) 235;

D. Choudhury, F. Eberlein, A. König, J. Louis and S. Pokorski, Phys. Lett. B342 (1995) 180.

[18] V. Kaplunovski and J. Louis, Phys. Lett. B306 (1993) 451;

R. Barbieri, J. Louis and M. Moretti, Phys. Lett. B312 (1993) 451;

J. Louis and Y. Nir, Munich preprint LMU-TPW 94-17, hep-ph/9411429.

[19] M. Dine, A. Kagan and S. Samuel, Phys. Lett. B243 (1990) 250;

M. Dine and A. Nelson, Phys. Rev. D48 (1993) 1277.

[20] M. Dine, A. Kagan and R. Leigh, Phys. Rev. D48 (1993) 4269.

[21] M. Leurer, Phys. Rev. Lett. 71 (1993) 1324; Phys. Rev. D49 (1994) 333.

[22] S. Davidson, D. Bailey and B.A. Campbell, Z. Phys. C61 (1994) 613.

[23] G. Bhattacharyya, J. Ellis and K. Srdihar, Phys. Lett. B336 (1994) 100, (E) B338 (1994) 522;

J.K. Mizukoshi, O.J.P. Eboli and M.C. Gonzalez-Garcia, CERN preprint CERN-TH7508-94, hep-ph/9411392. 